\title{
CTmod: a toolkit for Monte Carlo simulation of projections including scatter in computed tomography
}

\author{
Alexandr Malusek, Michael Sandborg and Gudrun Alm Carlsson
}

\section{Linköping University Post Print}

N.B.: When citing this work, cite the original article.

Original Publication:

Alexandr Malusek, Michael Sandborg and Gudrun Alm Carlsson, CTmod: a toolkit for Monte Carlo simulation of projections including scatter in computed tomography, 2008, Computer Methods and Programs in Biomedicine, (90), 2, 167-178.

http://dx.doi.org/10.1016/j.cmpb.2007.12.005

Copyright: Elsevier Science B.V., Amsterdam. http://www.elsevier.com/

Postprint available at: Linköping University Electronic Press

http://urn.kb.se/resolve?urn=urn:nbn:se:liu:diva-13035 


\title{
CTmod - a toolkit for Monte Carlo simulation of projections including scatter in computed tomography
}

\author{
Alexandr Malusek ${ }^{a, *}$ Michael Sandborga \\ Gudrun Alm Carlsson ${ }^{\text {a }}$ \\ ${ }^{a}$ Linköping University, Department of Radiation Physics, 58185 Linköping, \\ Sweden
}

\begin{abstract}
The CTmod toolkit is a set of $\mathrm{C}++$ class libraries based on the CERN's application development framework ROOT. It uses the Monte Carlo method to simulate energy imparted to a CT-scanner detector array. Photons with a given angle-energy distribution are emitted from the X-ray tube approximated by a point source, transported through a phantom, and their contribution to the energy imparted per unit surface area of each detector element is scored. Alternatively, the scored quantity may be the fluence, energy fluence, plane fluence, plane energy fluence, or kerma to air in the center of each detector element. Phantoms are constructed from homogenous solids or voxel arrays via overlapping. Implemented photon interactions (photoelectric effect, coherent scattering, and incoherent scattering) are restricted to the energy range from $10 \mathrm{keV}$ to $200 \mathrm{keV}$. Variance reduction techniques include the collision density estimator and survival biasing combined with the Russian roulette. The toolkit has been used to estimate the amount of scatter in cone beam computed tomography and planar radiography.
\end{abstract}

Key words: Monte Carlo, Computed tomography, Cone beam, Scatter

\section{Introduction}

In classical computed tomography (CT), useful information is carried to the detector array by primary photons, i.e. photons which have not interacted inside the phantom. Scattered photons add an undesirable signal which causes

* Corresponding author.

Email address: Alexandr.Malusek@imv.liu.se (Alexandr Malusek). 
the so called scatter artefact that manifests itself as cupping or streaks in reconstructed images $[1,2]$. The effect of scattered photons is especially strong in cone beam CT since the amount of scatter increases with increasing beam width. Other factors like the tube voltage, phantom size, collimators, bowtie filters, and detector array construction also play important roles. The ability to study how these factors affect primary and scatter projections helps in optimization of both the CT scanner design and image reconstruction algorithms.

The Monte Carlo (MC) method had been used to investigate the effect of scatter in planar radiography more than 20 years ago $[3,4]$ but only recently it finds its way to studies about the effect of scatter on reconstructed images in computed tomography, see for instance [5]. The need to calculate thousands of scatter projections in the order of hours still represents a challenge even for computer clusters and thus approximative methods are also being developed [6]. Our attempt to address the problem with scatter in cone beam CT was to develop a simulation toolkit that could calculate scatter projections using the MC method; primary projections were calculated analytically. The work started in 2001 and the main inspiration came from the work of Persliden [7] and from the Voxman code [8] (written in Fortran 77) which was used for calculations in planar radiography. The structure of the Voxman code was redesigned and based on the object-oriented data analysis framework ROOT [9] which uses the $\mathrm{C}++$ language. Features specific to CT-scanners like bowtie filters and sinograms were added. The toolkit was named CTmod. It was used, for instance, to calculate primary and scatter projections in cone beam CT [10] and planar radiography, and to evaluate the effect of scatter on reconstructed images [11]. A detailed description of its methods that would make the reproduction of our results possible had been missing. This article is supposed to fill the gap by providing an overview of methods used in the CTmod code; formulas and derivations that were too voluminous to be included here have been published in the report [12]. Many CTmod's features are not readily available in general purpose MC codes like EGS4, Geant4, MCNP, FLUKA, or Penelope [13-17]. Some of these features come with a price: the code is difficult to use. Nevertheless the authors believe that computational methods that prove their usefulness in CTmod will, sooner or later, be implemented in general purpose MC codes that are more suitable for production use.

\section{Computational methods}

CTmod uses concepts common to other Monte Carlo radiation transport codes. A history of a photon emitted from a source consists of a series of discrete interactions. The tracking in the geometry is performed as if the photon moved on line segments. Interactions are independent, i.e. an interaction is not affected by previous ones and depends only on the current photon's 
state. The state immediately after $i$ th interaction, $\alpha_{i}$, is given by four parameters $\alpha_{i}=\left(\mathbf{P}_{i}, \mathbf{u}_{i}, E_{i}, w_{i}\right)$, where $\mathbf{P}_{i}$ is the interaction point, $\mathbf{u}_{i}$ the photon's direction, $E_{i}$ its energy, and $w_{i}$ its (statistical) weight. A $j$ th history thus consists of a sequence of state points $\left(\alpha_{0}, \ldots, \alpha_{n_{j}}\right)$, where $\alpha_{0}$ describes the photon emitted from the source and $\alpha_{n_{j}}$ describes the event when the photon's history is terminated by the program.

\subsection{The Monte Carlo method}

Monte Carlo methods use random numbers to find solutions to mathematical problems. In our case, the method is used to calculate scatter projections by simulating photon transport inside a CT scanner. The transport can be simulated using analog or nonanalog methods; both approaches are available in CTmod. In the analog simulation, distributions of sampled random quantities correspond to real physical processes. In a nonanalog simulation, distributions of sampled quantities are modified to speed up the simulation by lowering variances of scored quantities without changing their mean values. The efficiency, $\varepsilon$, of a $\mathrm{MC}$ simulation can be defined as

$$
\varepsilon=[V(\bar{X}) t]^{-1}
$$

where $t$ is the computational time and $V(\bar{X})$ is the variance of the average of the scored quantity $X$. Note that since $V(\bar{X})$ is inversely proportional to the number of photon histories, the efficiency $\varepsilon$ is approximately constant for well behaved simulations. Variance reduction techniques implemented in CTmod include the collision density estimator, survival biasing, and Russian roulette. The collision density estimator, also known as point detectors [15], estimates the mean value of a point quantity (e.g. a particle fluence at a given point) by summing contributions from each photon interaction, see section 2.5. Survival biasing is a technique for extending the photon's life. If it is used, a photoelectric effect, which otherwise terminates the photon's history, only decreases photon's weight and the transport continues, see section 2.2.1. Russian roulette is a technique for removing particles with low statistical weight. The result of the game is either a killed particle or a particle with its weight increased by the factor $1 /(1-p)$, where $p$ is the user-adjustable probability that the particle is killed. It is used to avoid transport of photons that cannot significantly contribute to the scored quantity.

In CTmod, random quantities with known theoretical distributions are sampled using the inverse transform or acceptance-rejection methods, see for instance $[18,19]$. In the inverse transform method, a random number $\gamma$ is sampled from a uniform distribution in the interval $(0,1)$ and the sample $x$ of a random variable $\hat{x}$ is calculated as

$$
x=F^{-1}(\gamma),
$$


where $F^{-1}(\gamma)$ is the inverse of the cumulative distribution function of $\hat{x}$. For a discrete variable $\hat{x}$, this method reduces to finding an index $i$ of $x_{i}$ so that $\sum_{j=1}^{i} p_{j} \leq \gamma<\sum_{j=1}^{i+1} p_{j}$, where $p_{j}$ is the probability that $\hat{x}=x_{j}$.

The acceptance rejection method consists of several steps. First, a random number $x$ is sampled from a uniform distribution. Then, a second random number $\gamma$ is sampled. If $\gamma<f(x)$, where $f(x)$ is the probability density function (PDF) of $X$, then $x$ is accepted. Otherwise it is rejected and the algorithm is repeated. The efficiency of the acceptance rejection method depends on the shape of the PDF.

\subsection{Photon interactions}

CTmod simulates photon interactions in the energy range from 10 to $200 \mathrm{keV}$, namely coherent scattering, incoherent scattering and photoelectric effect. This energy range corresponds to x-ray tube voltages up to $200 \mathrm{kV}$; typical values in computed tomography for diagnostic purposes are between 80 and $140 \mathrm{kV}$.

Let $\Sigma_{\mathrm{In}}, \Sigma_{\mathrm{Co}}$, and $\Sigma_{\mathrm{Ph}}$ be the macroscopic cross sections of the incoherent scattering, coherent scattering, and photoelectric effect, respectively, for a given material and photon energy $E$. In CTmod, these are usually taken from the EPDL97[20] or XCOM[21] data libraries. Let $\Sigma_{\mathrm{t}}=\Sigma_{\mathrm{In}}+\Sigma_{\mathrm{Co}}+\Sigma_{\mathrm{Ph}}$ be the total cross section. If a photon interaction occurs then the ratios $p_{\text {In }}=\Sigma_{\text {In }} / \Sigma_{t}$, $p_{\mathrm{Co}}=\Sigma_{\mathrm{Co}} / \Sigma_{\mathrm{t}}$, and $p_{\mathrm{Ph}}=\Sigma_{\mathrm{Ph}} / \Sigma_{\mathrm{t}}$ give the probabilities of corresponding types of interactions. The sampling of the interaction type is performed using the direct method for a discrete random variable with (energy dependent) probabilities $p_{\text {In }}, p_{\mathrm{Co}}$, and $p_{\mathrm{Ph}}$.

Electrons liberated in photon interactions are considered locally absorbed and their transport is not simulated. Axial symmetry is supposed for all scattering interactions, polarization effects are neglected. The azimuthal angle $\phi$, figure 1 , is sampled uniformly from the interval $[0,2 \pi]$, the polar angle $\theta$ is sampled from the probability density function $(\mathrm{PDF}) p(\theta ; E)$ given as

$$
p(\theta ; E)=\frac{1}{\sigma(E)} \frac{\mathrm{d} \sigma(\theta ; E)}{\mathrm{d} \theta},
$$

where $\sigma$ is the total cross section of the scattering interaction, $\mathrm{d} \sigma / \mathrm{d} \theta$ is the corresponding differential cross section, and $E$ is the initial photon energy. 


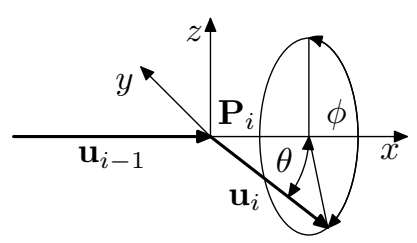

Fig. 1. The polar, $\theta$, and azimuthal, $\phi$, scattering angles. The x-axis of the local coordinate system $\left(\mathbf{P}_{i}, x, y, z\right)$ coincides with the original photon's direction $\mathbf{u}_{i-1}$. Note that $\mathbf{u}_{i}$ is the photon's direction after an interaction at the point $\mathbf{P}_{i}$

\subsubsection{Photoelectric Effect}

Two alternative approaches are available: the analog method, and the survival biasing method, sometimes also called analytical averaging of survival. In the analog method, the photon is absorbed and the history is terminated. In the biasing method, the new photon's weight $w_{i}$ is calculated as

$$
w_{i}=w_{i-1}\left(1-\sigma_{\mathrm{ph}} / \sigma_{\mathrm{t}}\right),
$$

where $\sigma_{\mathrm{ph}}$ and $\sigma_{\mathrm{t}}$ are the photoelectric and total cross sections respectively, and a coherent or incoherent scattering is simulated instead. Both the absorbed photon in the analog case and the captured weight $w_{i-1} \sigma_{\mathrm{ph}} / \sigma_{\mathrm{t}}$ in the survival biasing case may result in a photoelectron, Auger electron or characteristic radiation which is produced by electron transitions between shells. In CTmod, however, these emitted particles are assumed to be locally absorbed (the deposited energy is $E w_{i-1} \sigma_{\mathrm{ph}} / \sigma_{\mathrm{t}}$ ) and their transport is not simulated. This approach is acceptable for human tissues consisting of low $\mathrm{Z}$ elements.

\subsubsection{Coherent scattering}

The scattering angle $\theta$ of a photon with energy $E$ is sampled from the molecular differential cross section

$$
\frac{\mathrm{d} \sigma_{\mathrm{coh}}(\theta ; E)}{\mathrm{d} \theta}=\frac{r_{\mathrm{e}}^{2}}{2}\left(1+\cos ^{2} \theta\right) F_{m}^{2}(x) 2 \pi \sin \theta,
$$

where $x=E /(h c) \sin (\theta / 2)$ is a parameter related to the momentum transfer of the interaction $[22,23], F_{m}(x)$ is the molecular form factor of material with index $m, r_{\mathrm{e}}$ is the classical electron radius, $h$ is the Planck's constant, and $c$ is the speed of light. Persliden's combined method [7] is used: The parameter $x$ is obtained via the inverse method, the corresponding scattering angle $\theta$ is then tested for acceptance using the function $\left(1+\cos ^{2} \theta\right) / 2$. Detailed description of the algorithm is in [12].

If molecular form factors of a given material are not known then the free gas approximation, sometimes also called the independent atomic scattering hypothesis, is often used. In this approximation, the molecular cross section is 
calculated as a sum of cross sections of individual atoms. This rule is directly applicable to compounds where the number of atoms constituting a molecule is known. But for mixtures or body tissues where only the weight or atomic elemental fractions are known, the resulting sum cannot be related to a single molecule. In this case, we replace the molecular form factor $F_{m}(x)$ in (5) with its unnormalized version, $F_{m}^{*}(x)$, that can be calculated as [23]

$$
F_{m}^{* 2}(x)=\sum_{i} a_{i} F^{2}\left(x, Z_{i}\right)
$$

Here, $a_{i}$ is the atomic fraction of material with the atomic number $Z_{i}$. This replacement is possible since the corresponding PDF given by (3) is invariant to changes of the normalization factor. The free gas approximation may significantly overestimate the amount of photons scattered in the forward direction [24] and therefore the use of precise molecular form factors is preferred. Also, its usage may lead to errors in materials where wave interference affects the photon transport (e.g. in crystals).

In CTmod, the usage of form factors for a given material can be switched off, the scattering angle is then sampled from the Thompson cross section [7] which corresponds to $F(x)=1$ for all $x$. This approach is used for testing purposes only as it may lead to noticeable differences in the calculated amount of scatter. The differences result from the fact that the Thompson cross section is symmetric about $\pi / 2$ while the coherent scattering cross section is peaked forward, see figure 2 .

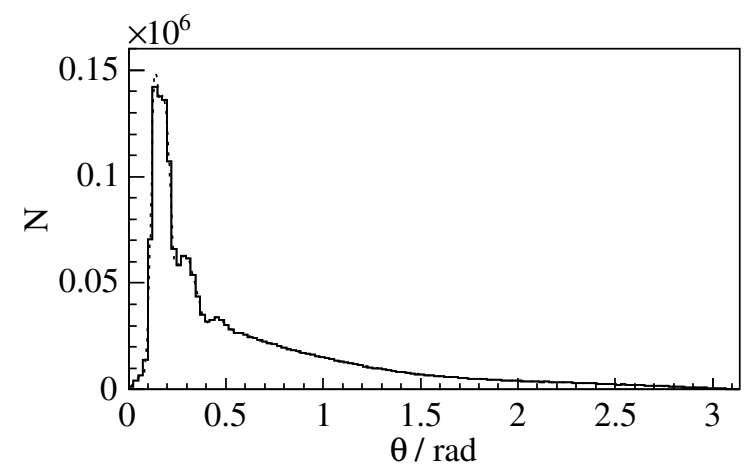

Fig. 2. Coherent scattering of $30 \mathrm{keV}$ photons in water: (-) The number of samples, $N$, with scattering angle $\theta$ in the corresponding bin. In total, $2 \times 10^{6}$ samples were taken. $(\cdots \cdots)$ The number of samples predicted from the theoretical PDF. Note that both curves almost overlap and that the scattering is peaked in the forward direction. 


\subsubsection{Incoherent scattering}

The scattering angle $\theta$ of a photon with energy $E$ is sampled from the molecular differential cross section

$$
\frac{\mathrm{d} \sigma_{\text {incoh }}(E, \theta)}{\mathrm{d} \theta}=\frac{r_{\mathrm{e}}^{2}}{2}\left(\frac{E^{\prime}}{E}\right)^{2}\left(\frac{E}{E^{\prime}}+\frac{E^{\prime}}{E}-\sin ^{2} \theta\right) S_{m}(x) 2 \pi \sin \theta,
$$

where $S_{m}(x)$ is the material-specific incoherent scattering function given by Hubbell [22], $r_{\mathrm{e}}$ is the classical electron radius, and $E^{\prime}$ is the energy of the scattered photon given by the Compton's formula

$$
E^{\prime}=E\left[1+E /\left(m_{\mathrm{e}} c^{2}\right)(1-\cos \theta)\right]^{-1} .
$$

Here, $m_{\mathrm{e}}$ is the electron rest mass, and $c$ is the speed of light. First, the energy $E^{\prime}$ is obtained from the Klein-Nishina differential cross section via a combined method described in [13]. Then the corresponding $\theta$ and $x$ are calculated and tested for acceptance using the function $S_{m}(x)$. Detailed description of the algorithm is in [12].

In the free gas approximation, we use the unnormalized incoherent scattering functions $S_{m}^{*}(x)$

$$
S_{m}^{*}(x)=\sum_{i} a_{i} S\left(x, Z_{i}\right)
$$

where $a_{i}$ is the atomic fraction of material with the atomic number $Z_{i}$, instead of the molecular versions $S_{m}(x)$. The replacement is possible since (3) does not depend on the normalization factor. The effect of the scattering function on the incoherent scattering differential cross section is less pronounced than the effect of form factors on coherent scattering; the free gas approximation is therefore often used. A histogram of scattering angles $\theta$ sampled from a distribution calculated for water from the free gas approximation is in figure 3 .

\subsection{The geometry}

CTmod contains its own geometry module; its core had been written before the more powerful ROOT geometry package [25] became available. The geometry is constructed from basic solids (spheres, ellipsoids, cylinders, boxes) and voxel arrays using an operation here referred to as overlapping. Basic solids must be convex and homogenous, voxel arrays are implemented as boxes consisting of equally sized voxels.

First, a solid-here referred to as the universe - is selected by the user as the base of the geometry. Other solids are then inserted into the geometry and their material replaces, or "overlaps", the original material of the solids 


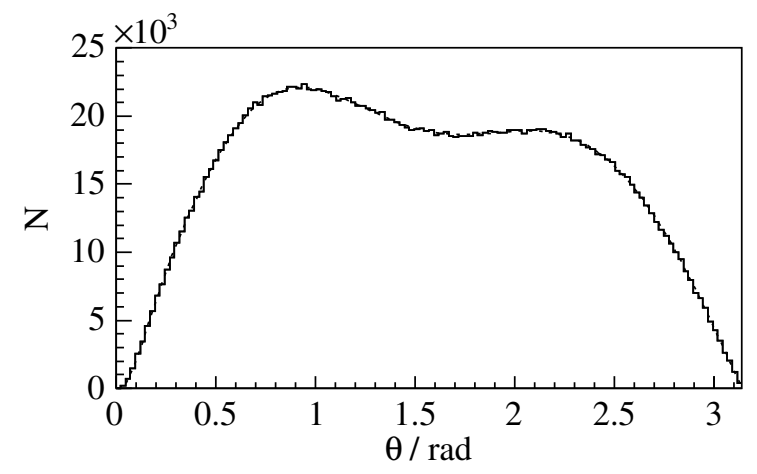

Fig. 3. Incoherent scattering of $50 \mathrm{keV}$ photons in water: (-) The number of samples, $N$, with scattering angle $\theta$ in the corresponding bin. In total, $2 \times 10^{6}$ samples were taken. $(\cdots \cdots)$ The number of samples predicted form the theoretical PDF. Note that both curves overlap.

that have already been inserted there. The order of insertion is important. The material at a given point of space is determined by the basic solid which contains the point and was inserted last.

Particle tracking is performed via a function which finds the current solid when the photon position is known, and via functions which determine the intersection of photon's trajectory with the nearest boundary. These intersections are found by solving linear or quadratic equations, see [12] for more information.

\subsubsection{Free and radiological path}

Consider a photon which moves along a line segment given via an initial point $\mathbf{P}$, direction $\mathbf{u}$, and distance $\lambda$. The probability, $P$, that a photon passes without an interaction is given by the well known formula $P=\exp \left(-o_{\lambda}\right)$ where the radiological path, $o_{\lambda}$, is given as the line integral

$$
o_{\lambda}=\int_{0}^{\lambda} \mu(\mathbf{P}+t \mathbf{u}) \mathrm{d} t .
$$

Here, $\mu$ is the linear attenuation coefficient; it is equal to the total macroscopic cross section, $\Sigma_{\mathrm{t}}$. The distance to the first interaction expressed via the radiological path, $o$, thus has en exponential distribution with the mean value equal to 1. In CTmod, it is sampled using the inverse transform method as

$$
o=-\ln \gamma,
$$

where $0<\gamma \leq 1$ is a random number with uniform distribution. The corresponding free path $\lambda$ of a particle in a homogenous solid $S$ is then calculated from (10) as

$$
\lambda(S, \mathbf{P}, \mathbf{u}, o)=o / \mu .
$$


In a voxel array, (10) simplifies to

$$
o=\sum_{i=0}^{n} \mu_{i} \Delta t_{i}
$$

where $\Delta t_{i}$ is the $i$ th line segment length of the trajectory, see figure 4 , and $n$ is the number of line segments. The free path is evaluated as a sum $\sum_{i=0}^{n} \Delta t_{i}$, where the lengths

$$
\Delta t_{i}=t_{i+1}-t_{i}, \quad i=0,1, \ldots, n-1
$$

are evaluated from distances $t_{i}$ between the point $\mathbf{P}$ and intersections of the ray with voxel-defining planes. Distances $t_{i}$ are calculated using a modified Siddon's algorithm [26]. In the Siddon's algorithm, the $\left\{t_{i}^{\mathrm{x}}\right\},\left\{t_{i}^{\mathrm{y}}\right\}$, and $\left\{t_{i}^{\mathrm{z}}\right\}$ arrays of equidistant values corresponding to intersections with voxel boundaries perpendicular to x-, y-, and z-directions, respectively, are calculated first and then merged and sorted to provide a single array $\left\{t_{i}\right\}$. The material corresponding to the interval $\left(t_{i}, t_{i+1}\right)$ is found by locating the voxel corresponding to the parameter $\left(t_{i}+t_{i+1}\right) / 2$. In our algorithm, the sorting of the $t_{i}$ values and the location of the next-entered voxel is performed on the fly as the photon is transported through the voxel array. In this case, extra care must be taken to ensure the stability of the algorithm, see [12] for more details. The length of the last segment $\Delta t_{n}$ is evaluated as

$$
\Delta t_{n}=\left(o-\sum_{i=0}^{n-1} \mu_{i} \Delta t_{i}\right) / \mu_{n}
$$

where $\mu_{n}$ is the linear attenuation coefficient of the voxel containing the final point.

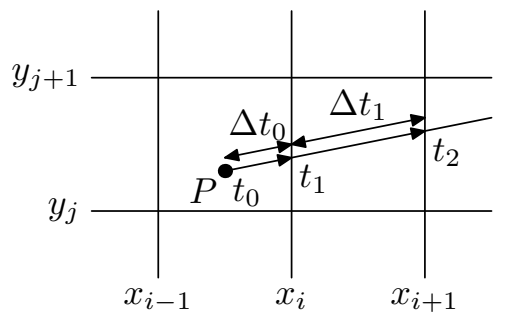

Fig. 4. Calculation of the free path in a voxel array.

\subsection{Photon sources}

Photon sources available in CTmod produce planar fan beams, cylindrical fan beams, and circular beams, see figure 5. Planar fan beams are used in CT scanners with flat panel detectors as they form a rectangular field at 
the detector's surface. Cylindrical fan beams are used in conventional third or fourth generation CT scanners where detector elements are located on a cylindrical surface. Circular beams are rarely used in CT practice but the user may benefit from their axial symmetry.

(a)

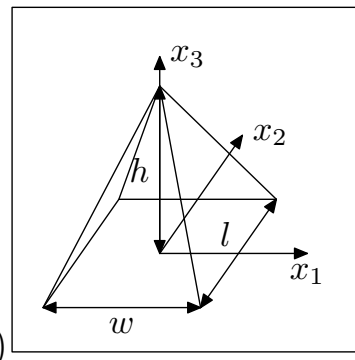

(b)

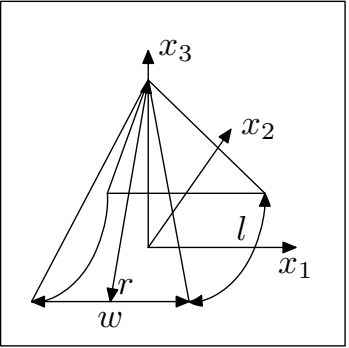

(c)

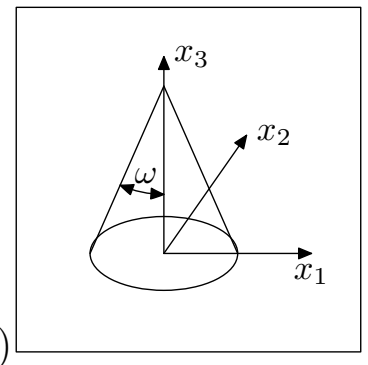

Fig. 5. (a) Planar fan beam: the projection of the collimated beam on a planar surface perpendicular to the beam axis is a rectangle. (b) Cylindrical fan beam: the projection on a cylindrical surface is a rectangle. (c) Circular beam: the projection on a planar surface is a full circle.

Let $s_{\Omega, \mathrm{E}}(\Omega, E) \mathrm{d} \Omega \mathrm{d} E$ be the number of photons with energy from $E$ to $E+\mathrm{d} E$ emitted into the solid angle $\mathrm{d} \Omega$ in the direction $\Omega$. In CTmod, photons are emitted from a single point and the angle-energy distribution $s_{\Omega, \mathrm{E}}(\Omega, E)$ can be written as

$$
s_{\Omega, \mathrm{E}}(\boldsymbol{\Omega}, E)=S s_{\Omega}(\boldsymbol{\Omega}) s_{\mathrm{E}}(E),
$$

where $s_{\Omega}(\Omega)=(4 \pi)^{-1} \mathrm{sr}^{-1}$ and $s_{\mathrm{E}}(E)$ are the directional and spectral probability density functions, respectively, and $S$ is the total number of emitted photons. All results are normalized to 1 photon emitted from the source, i.e. $S=1$. To increase efficiency, the code does not produce photons outside the beam. To account for this source biasing, the statistical weight of photons produced by the source is set to $w=\Delta \Omega /(4 \pi)$ for photons inside the beam and to $w=0$ otherwise. Solid angles $\Delta \Omega$ for beams in figure 5 are derived in [12]. The advantage of the normalization of scored quantities per 1 photon emitted into the solid angle of $4 \pi \mathrm{sr}$ is that their absolute values calculated for beams with varying width can be directly compared.

A direct sampling based on the inverse transform method is used to sample the initial photon's direction for the circular beam and the acceptance-rejection methods are used for the two fan beams. In the latter case, a position within the rectangle on the appropriate surface is sampled from two uniform distributions and tested for acceptance. Alternatively, the user can use the direct sampling method for the planar and cylindrical fan beams too but the acceptance-rejection method is often faster (for instance it was 1.2 times faster for configurations discussed in [12]). In CTmod, the energy spectrum of photons consists of continuous and discrete parts, the latter represents characteristic radiation, see figure 6 . To sample the energy of a photon, one of those two parts is randomly selected according to its probability. In the discrete part, 


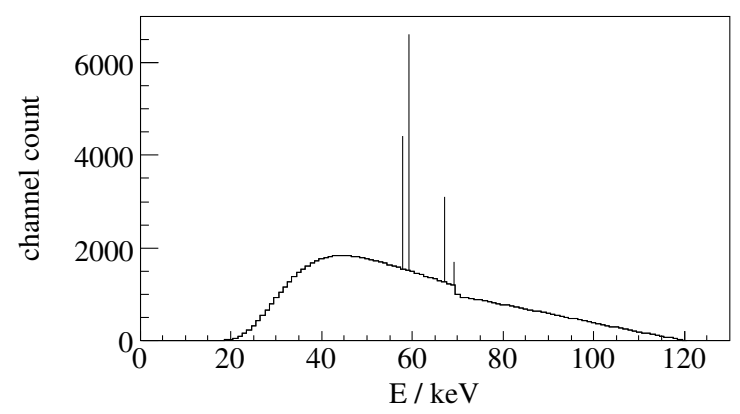

Fig. 6. An X-ray spectrum consists of a continuous part and discrete energy lines. Calculated using the Birch and Marshall code [29] for tube voltage $120 \mathrm{kV}, 16^{\circ}$ anode angle, tungsten anode, $2 \mathrm{~mm} \mathrm{Al}+0.1 \mathrm{~mm} \mathrm{Cu}$ filtration.

the energy is sampled according to the probability of each energy line using the direct sampling method. In the continuous part, the acceptance-rejection method is used. Though the efficiency of this method is relatively low, the computer spends only a small fraction of time in this routine. The Walker's method [27] is more efficient and may be implemented in the future. X-ray spectra can be taken from the catalogue [28], calculated using the Birch and Marshall code [29], or obtained - usually under a non-disclosure agreementfrom manufacturers for specific CT-scanner models.

An optional bowtie filter lowers the statistical weight of a photon according to its transmission function; photon interactions inside the bowtie filter are not simulated. Currently implemented bowtie filters are: a homogenous general cylinder whose thickness as a function of fan angle is taken from a file, a filter which fully compensates for a water cylinder phantom, and a set of simplified bowtie filters from commercial CT scanners.

\subsection{Scoring}

CTmod calculates projections, energy imparted to solids, and number of interactions in each solid.

Projections are calculated via a planar or cylindrical array of point detectors which score fluence, energy fluence, plane fluence, plane energy fluence, air collision kerma, or the energy imparted per unit surface area of a detector element, see [30] for definitions of these quantities. Let a radiation field be described by the angle-energy distribution of fluence, $\Phi_{\Omega, E}(\mathbf{r}, \Omega, E)$, where $\Phi_{\Omega, E}(\mathbf{r}, \Omega, E) \mathrm{d} A \mathrm{~d} E \mathrm{~d} \Omega$ is the number of photons with energy between $E$ and $E+\mathrm{d} E$ moving through the surface element $\mathrm{d} A$ perpendicular to a direction $\Omega$ at a point $\mathbf{r}$ into the element of the solid angle $\mathrm{d} \Omega$ in the direction $\Omega$. Then 
the fluence, $\Phi(\mathbf{r})$, at the point $\mathbf{r}$ is given as

$$
\Phi(\mathbf{r})=\int_{4 \pi} \int_{0}^{\infty} \Phi_{\Omega, E}(\mathbf{r}, \Omega, E) \mathrm{d} \Omega \mathrm{d} E
$$

and the energy fluence, $\Psi(\mathbf{r})$, as

$$
\Psi(\mathbf{r})=\int_{4 \pi} \int_{0}^{\infty} E \Phi_{\Omega, E}(\mathbf{r}, \Omega, E) \mathrm{d} \Omega \mathrm{d} E .
$$

These quantities describe the response of the so called thin photon counting and thin energy counting detectors, respectively. The word thin points out that these detectors attenuate impinging particles only negligibly. The plane fluence, $\Phi_{\mathbf{n}}(\mathbf{r})$, is given as

$$
\Phi_{\mathbf{n}}(\mathbf{r})=\int_{4 \pi} \int_{0}^{\infty} \Phi_{\Omega, E}(\mathbf{r}, \Omega, E) \boldsymbol{\Omega n} \mathrm{d} \Omega \mathrm{d} E,
$$

where $\mathbf{n}$ is the normal vector to the plane, and the plane energy fluence, $\Psi_{\mathbf{n}}(\mathbf{r})$, as

$$
\Psi_{\mathbf{n}}(\mathbf{r})=\int_{4 \pi} \int_{0}^{\infty} E \Phi_{\Omega, E}(\mathbf{r}, \boldsymbol{\Omega}, E) \boldsymbol{\Omega} \mathbf{n} \mathrm{d} \Omega \mathrm{d} E .
$$

These quantities describe the response of the so called thick photon counting and thick energy counting detectors, respectively. Thick detectors absorb almost all impinging particles. The air collision kerma, $K_{\mathrm{c}, \text { air }}(\mathbf{r})$, is given as

$$
K_{\mathrm{c}, \text { air }}(\mathbf{r})=\int_{4 \pi} \int_{0}^{\infty} E \Phi_{\Omega, E}(\mathbf{r}, \Omega, E)\left(\mu_{\mathrm{en}} / \rho\right)_{\text {air }} \mathrm{d} \Omega \mathrm{d} E,
$$

where $\left(\mu_{\mathrm{en}} / \rho\right)_{\text {air }}$ is the energy dependent mass energy-absorption coefficient [30]. At a point with charged particle equilibrium, the air collision kerma equals the absorbed dose to air. This fact is utilized by air ionization chambers.

The response of a real detector element can be described via the mean energy imparted to its sensitive volume. This quantity is proportional to the mean energy imparted per unit surface area of the detector element, $\bar{\epsilon}_{A}(\mathbf{r})$, which is given as

$$
\bar{\epsilon}_{A}(\mathbf{r})=\frac{\Delta \bar{\epsilon}}{\Delta A}=\int_{4 \pi} \int_{0}^{\infty} E \Phi_{\Omega, E}(\mathbf{r}, \Omega, E) \boldsymbol{\Omega} \mathbf{n} f(\mathbf{r}, E, \boldsymbol{\Omega}) \mathrm{d} \Omega \mathrm{d} E .
$$

Here, $\Delta \bar{\epsilon}$ is the mean energy imparted to a detector element with an associated reference area $\Delta A$ and $f(\mathbf{r}, E, \Omega)$ is the energy absorption efficiency function of the detector element (see section 2.5.3 for more details). Equation 22 can also be seen as the definition of the energy absorption efficiency function, its calculation is described in section 2.5.3.

To simplify the notation, the symbol $I$ is used for quantities defined in (17)(22) and the corresponding equations are written as

$$
I(\mathbf{r})=\int_{4 \pi} \int_{0}^{\infty} \Phi_{\Omega, E}(\mathbf{r}, \Omega, E) X \mathrm{~d} \Omega \mathrm{d} E
$$


where (i) $X=1$ for fluence, (ii) $X=E$ for energy fluence, (iii) $X=\boldsymbol{\Omega n}$ for plane fluence, (iv) $X=E \boldsymbol{\Omega} \mathbf{n}$ for plane energy fluence, (v) $X=E\left(\mu_{\mathrm{tr}} / \rho\right)_{\text {air }}$ for kerma to air, and (vi) $E \boldsymbol{\Omega} \mathbf{n} f(\mathbf{r}, E, \boldsymbol{\Omega})$ for the energy imparted per unit area. The unit of $I(\mathbf{r})$ depends on the definition of $X$ and may be e.g. $\mathrm{m}^{-2}, \mathrm{~J} \mathrm{~m}^{-2}$ or Gy.

Energy imparted to each solid is calculated by scoring the energy imparted in each interaction. This quantity can also be calculated for each voxel of a voxel array and CTmod can then report the average absorbed dose to each voxel or the effective dose to an anthropomorphic phantom if corresponding tissue weighting factors were provided by the user.

\subsubsection{Primary projection}

Primary projection, $I_{\mathrm{p}}\left(\mathbf{r}_{\mathrm{d}}\right)$ is calculated by numerical integration of the formula

$$
I_{\mathrm{p}}\left(\mathbf{r}_{\mathrm{d}}\right)=\int_{0}^{\infty} \frac{s_{\Omega, \mathrm{E}}\left(\boldsymbol{\Omega}_{\mathrm{d}}, E\right) w}{d^{2}} \exp \left[-\int_{0}^{d} \mu\left(E, \mathbf{r}_{\mathrm{s}}+t \boldsymbol{\Omega}_{\mathrm{d}}\right) \mathrm{d} t\right] X \mathrm{~d} E
$$

where $d$ is the the distance between the source and point detector positions $\mathbf{r}_{\mathrm{s}}$ and $\mathbf{r}_{\mathrm{d}}$, respectively, $s_{\Omega, \mathrm{E}}$ is the source intensity, $\mu$ is the linear attenuation coefficient, $\Omega_{\mathrm{d}}$ is the direction from the source to the point detector, and $X$ is defined in connection to (23). The statistical weight $w$ is defined in connection to $(16)$.

\subsubsection{Scatter projection}

The collision density estimator is used to calculate quantities defined in (17)(22). Each scattering interaction contributes

$$
\Delta X_{i}=\frac{p\left(\boldsymbol{\Omega}_{i-1}, \boldsymbol{\Omega}_{\mathrm{d}}\right)}{4 \pi d^{2}} \exp \left[-\lambda\left(E_{i}, \mathbf{r}_{\mathrm{d}}, \mathbf{r}_{i}\right)\right] w_{i} X
$$

to a point detector at position $\mathbf{r}_{\mathrm{d}}$. Here, $\left(\mathbf{r}_{i}, \boldsymbol{\Omega}_{i}, E_{i}, w_{i}\right)$ is the photon state after $i$ th interaction, $d$ is the distance between the point detector and the interaction point, $\lambda$ is the radiological path, and $p\left(\boldsymbol{\Omega}_{i-1}, \boldsymbol{\Omega}_{\mathrm{d}}\right)$ is the PDF of scattering from the direction $\Omega_{i-1}$ to $\Omega_{\mathrm{d}}$. An example of a photon history is in figure $7 \mathrm{~b}$. It consists of en emission from the X-ray source, an incoherent scattering, a coherent scattering, and a photoelectric effect which terminates the history. 

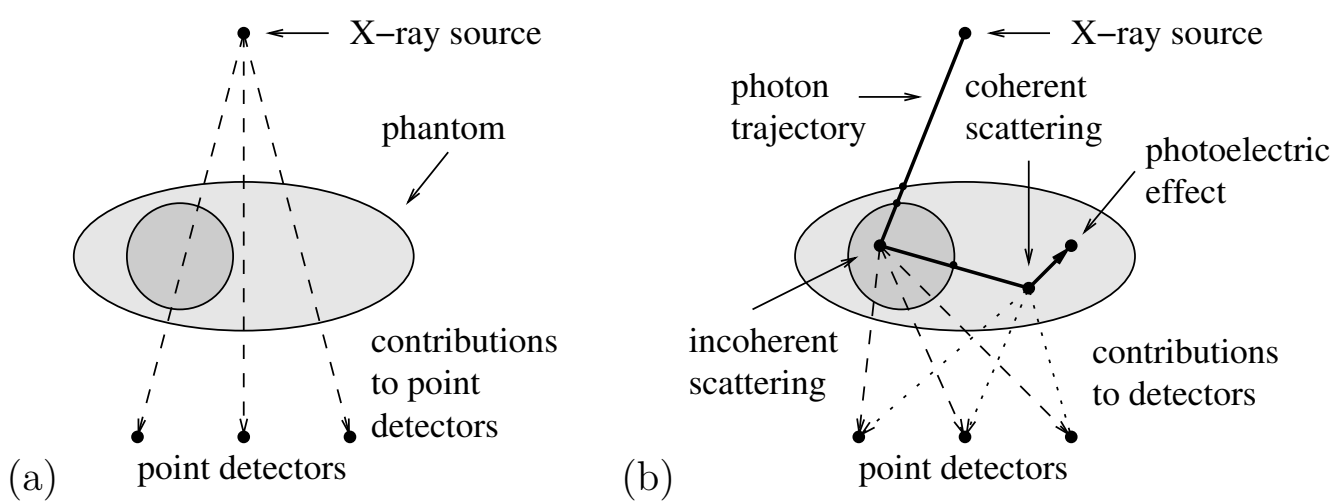

Fig. 7. (a) To calculate a primary projection, line integrals from the source to each point detector are evaluated. (b) To calculate a scatter projection, the history of a photon is simulated and each point detector registers contributions corresponding to line integrals from every scattering interaction.

The mean value of the scored quantity per one history, $\bar{I}_{\mathrm{h}, \mathrm{s}}$, is estimated as

$$
\bar{I}_{\mathrm{h}, \mathrm{s}}=\frac{1}{N} \sum_{i=1}^{N} \Delta X_{\mathrm{h}, i},
$$

where $N$ is the number of histories and $\Delta X_{\mathrm{h}, i}$ is the contribution from all scattering interactions in history $i$. The subscripts "s" and " $\mathrm{h}$ " in $\bar{I}_{\mathrm{h}, \mathrm{s}}$ stand for "scatter" and "history", respectively. The variance of the average is estimated as

$$
\sigma_{\bar{I}_{\mathrm{h}, \mathrm{s}}}^{2}=\frac{1}{N(N-1)} \sum_{i=1}^{N}\left(\Delta X_{\mathrm{h}, i}-\bar{I}_{\mathrm{h}, \mathrm{s}}\right)^{2} .
$$

Sums in (26) and (27) are calculated via counters $\sum \Delta X_{\mathrm{h}, i}$ and $\sum \Delta X_{\mathrm{h}, i}^{2}$ which are updated according to the method described by Sempau [31].

Besides the intensity values, CTmod can produce: (i) a particle event data file which stores photon's kinematic data for each interaction, (ii) detector event data files which store information about photons contributing to the signal of corresponding point detectors, and (iii) a figure of merit event file which can be used for convergence speed analysis.

\subsubsection{Energy absorption efficiency function of a detector element}

Consider an infinite array of detector elements (a repeated structure in MCNP), see figure 8 , and a photon field expanded from a point $\mathbf{r}$ to the whole space (see [32] for more information about the concept of an expanded field). An expanded field can be treated as a superposition of monoenergetic broad parallel beams so we restrict the following discussion to a broad parallel beam of photons with energy $E$ and direction of flight $\Omega$. For such a field, the energy absorption efficiency function, $f(\mathbf{r}, E, \boldsymbol{\Omega})$, of a detector element is a non-stochastic quantity that gives the fraction of energy carried by photons 
impinging on the entrance surface of the detector element that is imparted to its active volume. It combines the quantum absorption efficiency and the geometric efficiency of the detector element. In [33], we showed that (i) the mean energy imparted to the active volume of a detector element by a field expanded to the whole space equals the mean energy imparted to active volumes of all detector elements by a field expanded to the entrance area of the detector element, and (ii) the reference area-defined as a shifted entrance area - can be positioned at any depth of the detector element. Because of this, the calculation of $f(\mathbf{r}, E, \boldsymbol{\Omega})$ can be performed as follows. The vicinity of the detector element is modeled by a sufficiently large array of detector elements (see figure 8) and a reference area is selected at any depth of the detector element. For detector elements with a collimator like in figure 8, the reference

(a)
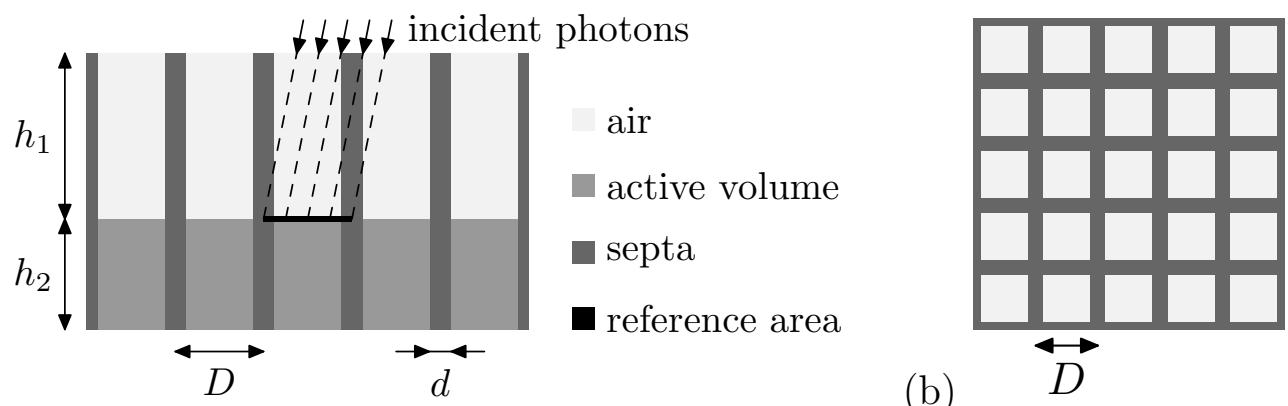

Fig. 8. Side view (a) and top view (b) of a $5 \times 5$ array with equally sized detector elements. Dimensions used to calculate the energy absorption efficiency function in figure 9 were: detector element width $D=1 \mathrm{~mm}$, septa width $d=0.1 \mathrm{~mm}$, air height $h_{1}=5 \mathrm{~mm}$, and active volume height $h_{2}=3 \mathrm{~mm}$.

area can, for instance, coincide with the entrance area of the active volume. Initial photon positions are sampled by sampling positions at the reference area from a uniform distribution and back-projecting corresponding photon trajectories on the array's surface, see figure 8. The energy imparted to all active volumes is then simulated using a Monte Carlo code. Examples for an infinite slab and a detector array with and without a collimator are in [33].

The broad parallel beam of photons with energy $E$ and direction $\Omega$ can be simulated using $N$ photons impinging at the entrance area $\Delta A$. The corresponding angle-energy distribution of fluence, $\Phi_{\Omega, E}(\Omega, E)$, is then

$$
\Phi_{\Omega, E}(\boldsymbol{\Omega}, E)=\frac{N}{\Delta A|\mathbf{\Omega n}|} \delta\left(\boldsymbol{\Omega}-\boldsymbol{\Omega}_{0}\right) \delta\left(E-E_{0}\right)
$$

where $\delta$ is the Dirac's function and $\mathbf{n}$ is the normal to the entrance area. The mean energy imparted to the active volume of all detector elements is then 


$$
\begin{aligned}
\bar{\epsilon} & =\int_{\Delta A} \int_{4 \pi} \int_{0}^{\infty} E \Phi_{\Omega, E}(\Omega, E)|\mathbf{\Omega}| f(E, \Omega) \mathrm{d} A \mathrm{~d} \Omega \mathrm{d} E \\
& =\int_{\Delta A} E_{0} \frac{N}{\Delta A} f\left(E_{0}, \Omega_{0}\right) \mathrm{d} A \\
& =E_{0} N f\left(E_{0}, \Omega_{0}\right)
\end{aligned}
$$

From 31, the energy absorption efficiency function can be calculated as

$$
f\left(E_{0}, \Omega_{0}\right)=\frac{\bar{\epsilon}}{E_{0} N}
$$

If the detector element is approximated by an infinite slab then the shift invariance of the geometry simplifies the simulation so that a pencil beam can be used. For screen-film systems, this quantity is referred to as the average fractional energy, $P_{E}$, by Chan [34].

Implementation in CTmod considers the dependence on the photon energy, $E$, and the cosine of the incidence angle, $\xi=\cos \theta$, only. Table of $f(E, \xi)$ values is pre-calculated using the MCNP [15] or Penelope [17] codes at a grid of equidistant points. Typically, $E$ ranges from 1 to $150 \mathrm{keV}$ with a $1 \mathrm{keV}$ step and $\xi$ ranges from 0 to 1 with a $1 / 64$ step. Bilinear interpolation [35] is used to obtain functional values at inner points and linear extrapolation is used for $\xi$ between 0 and $1 / 64$ to avoid the discontinuity of $f(E, \xi)$ at $\xi=0$, which corresponds to photons impinging parallel to the detector surface.

An ideal detector absorbs all energy of an impinging photon and thus its energy absorption efficiency function is $f(E, \boldsymbol{\Omega})=1$. In a conventional CT, the thickness of a solid state scintillator is selected so that it stops at least $95 \%$ photons in the energy range from 30 to $120 \mathrm{keV}$ [36]. An example of a highly absorbing detector element is a $3 \mathrm{~mm}$ thick infinite slab of the ceramic scintillator $\mathrm{Y}_{1.34} \mathrm{Gd}_{0.6} \mathrm{Eu}_{0.06} \mathrm{O}_{3}[36,37]$, also known as $(\mathrm{Y}, \mathrm{Gd})_{2} \mathrm{O}_{3}: \mathrm{Eu}$, whose energy absorption efficiency function for perpendicularly impinging photons is in figure 9. Note that discontinuities at energy levels of K and L-edges of gadolinium and yttrium are due to the escape of characteristic radiation which does not contribute to the energy imparted to the detector. In practice, detector elements are separated by septa which decreases the amount of scatter by attenuating obliquely incident photons but, on the other hand, it also decreases the geometric efficiency of the detector. For a detector element in figure 8, the geometric efficiency is $0.9^{2} / 1.0^{2}=0.81$ and the simulated energy absorption efficiency function is in figure 9. Values of the energy absorption efficiency function may be noticeably lower than 1 for a general-purpose detector array, e.g. a flat panel imager with a $600 \mu \mathrm{m}$ infinite layer of CsI:Tl, see figure 9.

Finally, we note that (i) the mean energy imparted to the active volume of the detector element with indices $(i, j)$, i.e. $\bar{\epsilon}_{i, j}$, by a broad parallel beam of photons impinging on the surface of the detector element $(0,0)$ represents the 


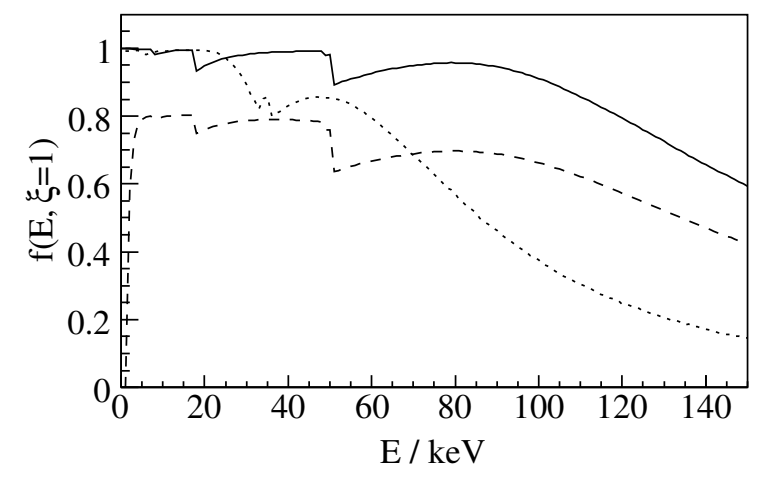

Fig. 9. Energy absorption efficiency, $f(E, \xi=1)$, of an infinite $3 \mathrm{~mm}$ slab of $(\mathrm{Y}, \mathrm{Gd})_{2} \mathrm{O}_{3}: \mathrm{Eu}(-)$, a detector element made of $(\mathrm{Y}, \mathrm{Gd})_{2} \mathrm{O}_{3}: \mathrm{Eu}$ and tantalum septa according to figure $8(---)$, and $600 \mu \mathrm{m}$ infinite slab of CsI:Tl $(\ldots \cdots)$ as a function of photon energy, $E$, for perpendicularly impinging photons. Statistical error on the $95 \%$ confidence level is lower than $1 \%$.

cross-talk between the two detector elements, and (ii) the scoring scheme used in CTmod can be extended to take this cross talk into account. See [33] for more information.

\subsubsection{Antiscatter grid}

The energy absorption efficiency function of a detector element may be multiplied by the analytical transmission formula of a linear antiscatter grid, see figure 10. This corresponds to a situation when the antiscatter grid is located in front of the detector element and the amount of scatter and characteristic radiation produced in the grid is negligible. The transmission formula was implemented according to Day [38]. The user may define the grid orientation for the whole point detector array or for each detector element.

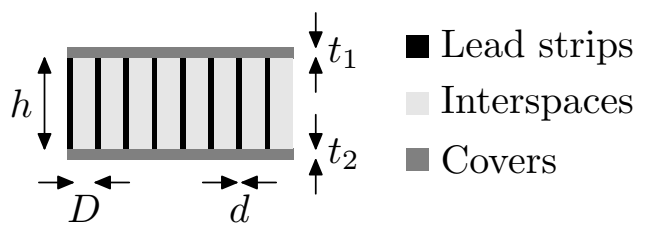

Fig. 10. A layout of a linear antiscatter grid with the strip width $d$, interspace width $D$, upper and lower cover thicknesses $t_{1}$ and $t_{2}$, respectively, and the strip height $h$.

\section{Program description}

The CTmod toolkit is implemented as a set of $\mathrm{C}++$ class libraries based on the CERN's application development framework ROOT [9]. The CTmod 
library performs simulations of photon transport, the AmEpdl97 library prepares cross section data.

\subsection{Program flow}

The main loop that controls the simulation of photon histories generates a photon from the source and starts the simulation of its transport. This is repeated many times, typically $10^{7}$. The simulation of individual histories is described in a simplified way by the following piece of code (also look at the notes at the end):

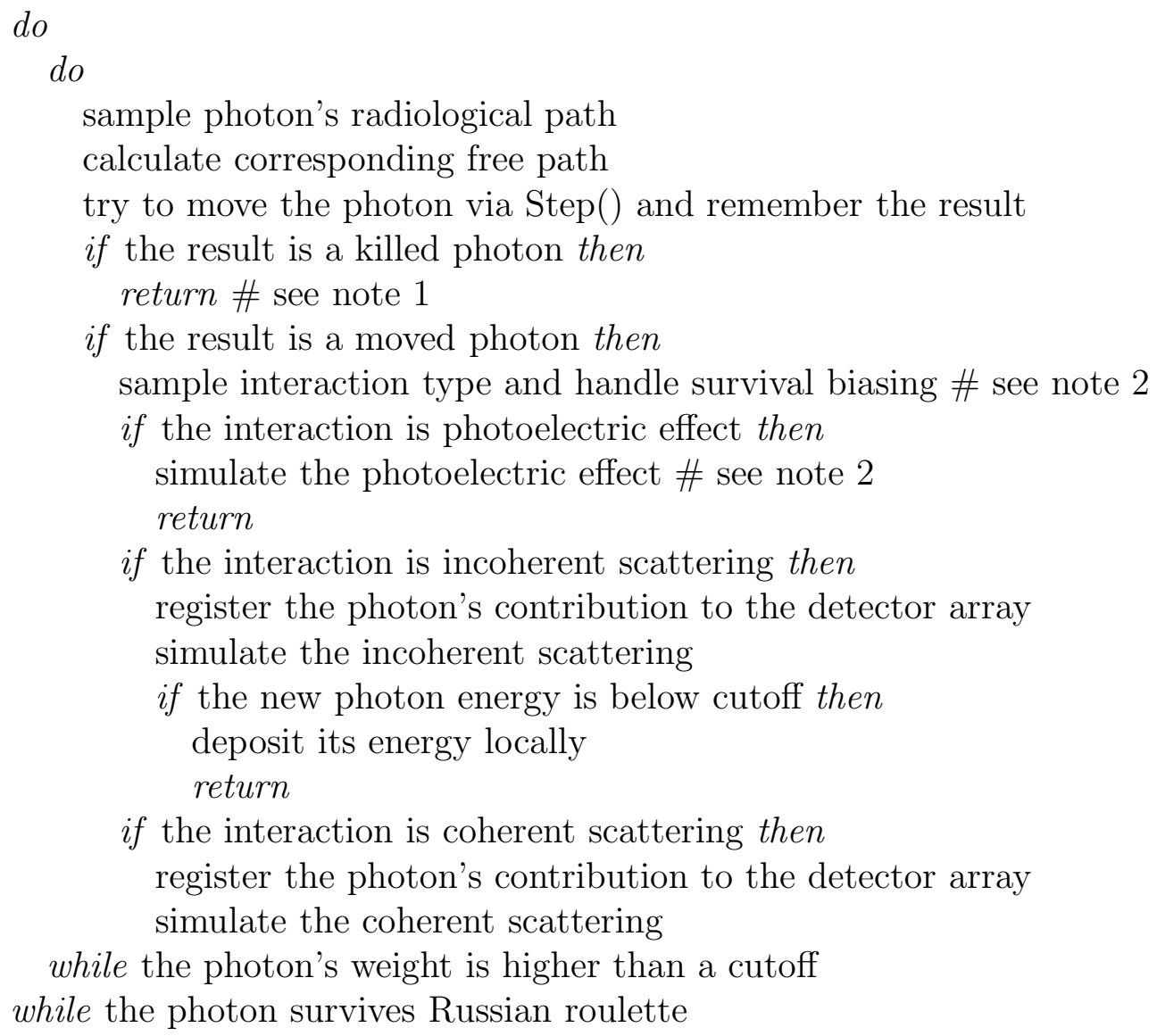

if the interaction is coherent scattering then register the photon's contribution to the detector array simulate the coherent scattering

while the photon's weight is higher than a cutoff while the photon survives Russian roulette

Note 1: Photons are killed when they leave the geometry. Note 2: If survival biasing is used then only incoherent and coherent scattering interactions are sampled, the photon's weight is adjusted by the sampling routine according to (4) and the corresponding energy is deposited locally.

The routine $\operatorname{Step}()$ transports the photon in the geometry. It tests for intersections of the photon path with solid boundaries and moves the photon to the intersection or, if no intersection is found, it transports the photon over 
the free path in the current solid:

for each solid in the overlapping solids list do

if the line segment intersects the solid then

if the distance is the smallest one then

remember the solid

done

if the line segment intersected an overlapping solid then

move the particle to the intersection point

return

if the particle leaves the current solid then

if the solid is the universe then

mark the photon as killed

return

for each solid in the base solid list do

if the new point is the base solid then

move the particle to the new point

return

done

move the photon \# the photon stays in the current solid

\section{Samples of program runs}

The capabilities of the CTmod toolkit are demonstrated on the simulation of primary and scatter projection profiles of a water cylinder. The setup consisted of a point source, a phantom, and an array of point detectors. The point source emitted monoenergetic photons with energies 30,90 , and $120 \mathrm{keV}$, respectively, into a cylindrical fan beam with the radius $R=1 \mathrm{~m}$, width $w=20 \mathrm{~mm}$, and length $l=900 \mathrm{~mm}$, the source-axis distance was $H=700 \mathrm{~mm}$, figure 11 . The phantom was a homogenous water cylinder with diameter $160 \mathrm{~mm}$ and

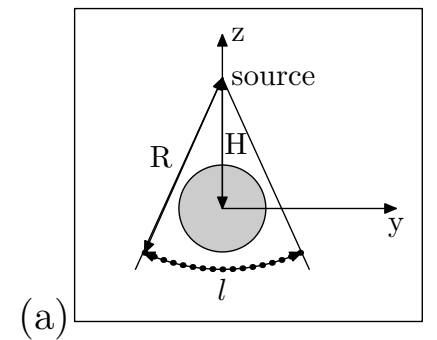

(b)

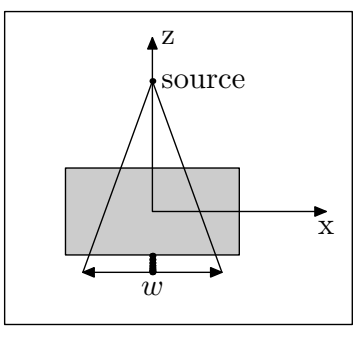

Fig. 11. Front view (a), and side view (b) of the CT geometry. The source-detector distance was $R=1 \mathrm{~m}$, the source-axis distance was $H=700 \mathrm{~mm}$ and the detector array length was $l=900 \mathrm{~mm}$.

height $250 \mathrm{~mm}$ located at the iso-center. The cylindrical point detector array consisted of 128 elements, the angular distance between any two adjacent point 
detectors was approximately $7.1 \times 10^{-3} \mathrm{rad}$. It corresponded to a detector spacing of $7.1 \mathrm{~mm}$. The energy absorption efficiency function of a $3 \mathrm{~mm}$ slab of the ceramic scintillator $(\mathrm{Y}, \mathrm{Gd})_{2} \mathrm{O}_{3}:$ Eu was used, see figure 9 , an antiscatter grid was not present. Cross sections and incoherent scattering functions were obtained from the EPDL97 data library. Molecular form factors for water were obtained from Morin [39].

Figure 12 shows the the estimate of mean energy imparted per unit area to a detector element $i$ by primary, $I_{\mathrm{p}}(i)$, and scattered, $I_{\mathrm{s}}(i)$, photons. The scatter to primary ratio $R_{\mathrm{sp}}(i)=I_{\mathrm{s}}(i) / I_{\mathrm{p}}(i)$ is also plotted. Values correspond to one photon emitted from the source into the solid angle of $4 \pi$ sr. Note that the
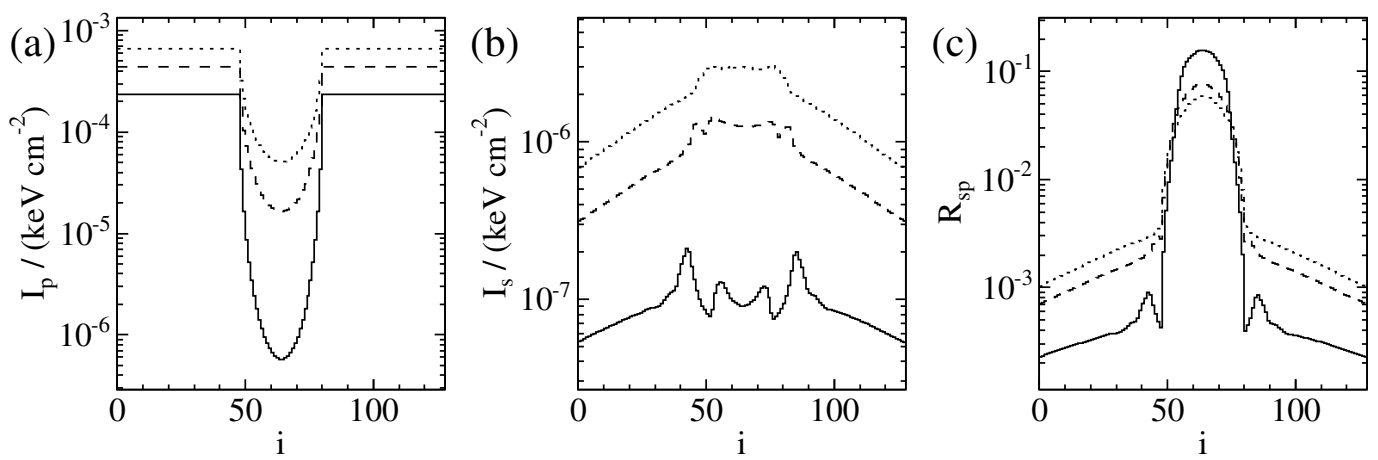

Fig. 12. Profiles of the mean energy imparted per unit area of a a detector element for (a) primary, (b) scatter, and (c) scatter to primary ratio for $30 \mathrm{keV}(-), 60 \mathrm{keV}$ (- - - -), and $120 \mathrm{keV}(\ldots \ldots)$ ) photons. Each quantity is plotted as a function of the point detector index $i$. Statistical error on the $95 \%$ confidence level was less than $1 \%$.

oscillatory shape of the scatter profile is due to a complex dependence of the molecular form factor of water on the parameter $x$, see (2.2.2). Also note that the function $I_{\mathrm{s}}$ changes significantly less with position than the function $I_{\mathrm{p}}$ and therefore $R_{\mathrm{sp}}$ mirrors the function $I_{\mathrm{p}}$.

Figure 13 shows the percentage of photon histories as a function of the number of coherent and incoherent scattering interactions that occurred during a single history; these were obtained from the optional particle event data file for the source emitting $30 \mathrm{keV}$ photons. The fraction of histories that ended without any scattering interaction (the photon was absorbed by the photoelectric effect or left the geometry) dominated (42\%) followed by the fraction of histories with one incoherent scattering interaction (20\%). In this particular case, the number of histories that ended without any coherent scattering interaction, $n_{\mathrm{Co}}=0$, decreased almost exponentially as a function of the number of incoherent scattering interactions, $n_{\mathrm{In}}$. 


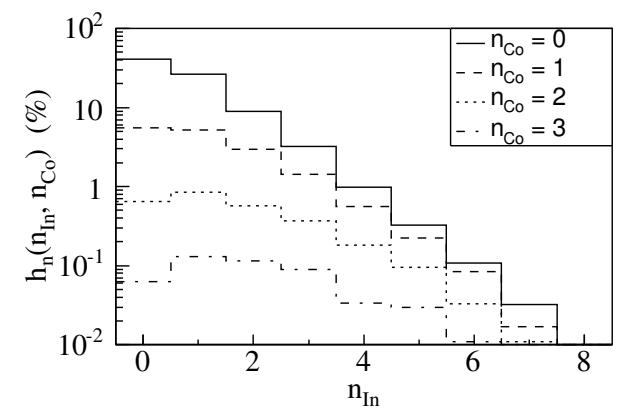

Fig. 13. The percentage of histories, $h_{\mathrm{n}}\left(n_{\mathrm{In}}, n_{\mathrm{Co}}\right)$, as a function of the number of incoherent, $n_{\mathrm{In}}$, and coherent scattering interactions, $n_{\mathrm{Co}}$, in a single history. The source emitted $30 \mathrm{keV}$ photons.

\section{$5 \quad$ Validation and verification}

To validate the CTmod toolkit, i.e. to make sure that codes based on the toolkit are applicable in practice, scatter-to-primary ratios of exposure in [40] were compared to data simulated using a CTmod based code. A notable discrepancy was found, see [41]. We suspect that extra-focal radiation and scatter from collimators and walls affected the experimental data; the issue has not been resolved yet. As an alternative, a comparison with the MCNP5 code [15] was performed, see [41]. The energy fluence by primary and scattered photons was calculated using MCNP5 at 12 points in the geometry in figure 11. Two phantoms of different sizes were used: (i) a head-size cylinder with the diameter $16 \mathrm{~cm}$ and height $25 \mathrm{~cm}$ and (ii) a body-size cylinder with the diameter $32 \mathrm{~cm}$ and height $50 \mathrm{~cm}$. The point isotropic source emitting photons with energy $30,60,90$, or $120 \mathrm{keV}$ was surrounded by two totally absorbing cylinders to create a cylindrical fan beam. No statistically significant difference between the two codes was found, see [41] for more information. A subset of calculated data is in figure 14 .

(a)

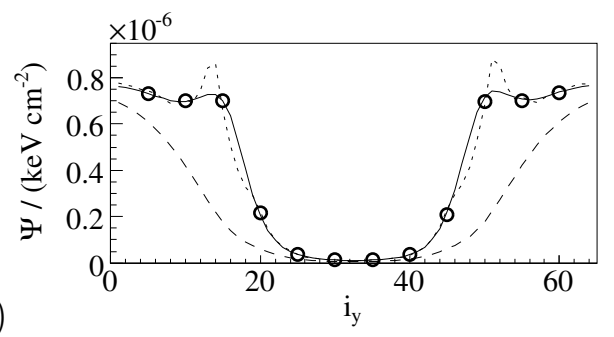

(b)

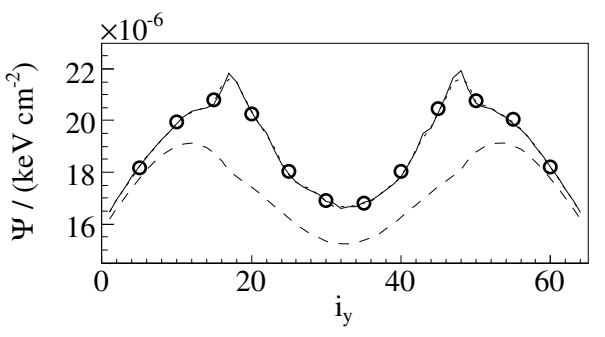

Fig. 14. Scatter projection profiles of the water cylinder with the radius of $16 \mathrm{~cm}$ and height of $50 \mathrm{~cm}$. Energy fluence, $\Psi$, of scattered photons is plotted as a function of detector index for initial photon energies of $30 \mathrm{keV}$ (a) and $120 \mathrm{keV}$ (b). Data were calculated using MCNP5 (o), CTmod with atomic form factors (-), CTmod with molecular form factors $(\cdots \cdots)$, and CTmod with coherent scattering approximated with the Thompson cross section $(---)$. 
To verify the CTmod toolkit, i.e. to make sure the software is coded correctly, test runs were performed. These were especially important for features that could not be tested against MCNP5 like bowtie filters, detector response functions, antiscatter grids, and voxel arrays.

\section{Program availability and supported platforms}

The CTmod library is available to its authors and to collaborating researchers only. Currently, there are no plans for its public release. The reasons range from unresolved licensing issues to a lack of man power to support external users. The AmEpd197 library is available under GNU GPLv2 from the authors.

The CTmod and AmEpdl97 libraries are written in ISO compliant C++. CTmod uses POSIX alarm signal handling for the prediction of job duration which may cause compilation problems on some platforms. The development has been performed on Linux (x86) with various versions of the ROOT application development framework, GNU Compiler Collection (GCC), and Intel $\mathrm{C}++$ compiler.

\section{Acknowledgments}

The authors are grateful for the helpful comments and suggestions given by Dr. Maria Magnusson, Linköping University, in the preparation of this paper and to Dr. David Dance, The Royal Marsden Hospital, United Kingdom, for sharing his information about the Voxman code. This work was supported by grants from the Swedish Research Council (VR: 621-2003-4648), from the Swedish Foundation for Strategic Research (R98:006) and conducted within the Center for Medical Image Science and Visualization (CMIV) at Linköping University.

\section{References}

[1] P.M. Joseph and R.D. Spital, The effects of scatter in x-ray computed tomography, Med. Phys. 9 (1982) 464-472

[2] G.H. Glover, Compton scatter effects in CT reconstructions, Med. Phys. 9 (1982) 860-867

[3] W. Kalender, Monte Carlo calculations of x-ray scatter data for diagnostic radiology, Phys. Med. Biol. 26 (1981) 835-849. 
[4] H.P. Chan and K. Doi, Physical characteristics of scattered radiation in diagnostic radiology: Monte Carlo simulation studies, Med. Phys. 12 (1985) $152-165$.

[5] W. Zbijewski and F.J. Beekman, Efficient Monte Carlo based scatter artifact reduction in cone-beam micro-CT, IEEE Trans. Med. Imaging 25 (2006) 817827.

[6] Y. Kyriakou, T. Riedel and W.A. Kalender, Combining deterministic and Monte Carlo calculations for fast estimation of scatter intensities in CT, Phys. Med. Biol. 51 (2006) 4567-4586.

[7] J. Persliden, A Monte Carlo program for photon transport using analogue sampling of scattering angle in coherent and incoherent scattering processes, Comput. Programs Biomed. 17 (1983) 115-128.

[8] M. Sandborg, D.R. Dance, J. Persliden and G. Alm Carlsson, A Monte Carlo program for the calculation of contrast, noise and absorbed dose in diagnostic radiology, Comput. Methods Programs Biomed. 42 (1994) 167-180.

[9] R. Brun and F. Rademakers, ROOT - an object oriented data analysis framework, Nucl. Inst. and Meth. in Phys. Res. A 389 (1997) 81-86. See also http://root.cern.ch/.

[10] A. Malusek, M. Sandborg, G. Alm Carlsson, Simulation of scatter in cone beam CT-effects on projection image quality, Proc. SPIE 5030 (2003) 740-751.

[11] A. Malusek, M.M. Seger, M. Sandborg and G. Alm Carlsson, Effect of scatter on reconstructed image quality in cone beam computed tomography: evaluation of a scatter-reduction optimisation function, Radiat. Prot. Dosimetry, 114 (2005) $337-340$.

[12] A. Malusek, M. Sandborg and G. Alm Carlsson, CTmod - mathematical foundations, ISRN ULI-RAD-R-102-SE, 2007. Available on http://huweb.hu.liu.se/inst/imv/radiofysik/publi/rap.html.

[13] W.R. Nelson, H. Hirayama and D.W.O. Rogers, The EGS4 code system, SLACReport-265, Stanford Linear Accelerator Center, Stanford University, 1985.

[14] S. Agostinelli et al., GEANT4 - a simulation toolkit, Nucl. Inst. and Meth. in Phys. Res. A 506 (2003) 250-303.

[15] X-5 Monte Carlo Team, MCNP - a general Monte Carlo n-particle transport code, version 5, LA-UR-03-1987, Los Alamos National Laboratory, 2004.

[16] A. Fassó, A. Ferrari, J. Ranft and P.R. Sala, FLUKA: a multi-particle transport code, CERN-2005-10 (2005), INFN/TC_05/11, SLAC-R-773

[17] J. Baro, J. Sempau, J. M. Fernandez-Varea and F. Salvat, PENELOPE: an algorithm for Monte Carlo simulation of the penetration and energy loss of electrons and positrons in matter, Nucl. Inst. and Meth. in Phys. Res. B 100 (1995) 31-46. 
[18] L. Montanet et al., Review of particle properties, Physical Review D, 50 (1994) $1173-1814$.

[19] J.M. Hammersley and D.C. Handscomb, Monte Carlo methods, (Chapman and Hall, London \& New York, 1964)

[20] D.E. Cullen, J.H. Hubbell and L. Kissel, EPDL97: the evaluated photon data library, '97 version, UCRL-50400 Vol. 6, Rev. 5, Lawrence Livermore National Laboratory, September 1997.

[21] M.J. Berger, J.H. Hubbell, S.M. Seltzer,J. Chang, J.S. Coursey, R. Sukumar and D.S Zucker, XCOM: Photon Cross Section Database (version 1.3), 2005, Available on: http://physics.nist.gov/xcom [2007, July 4], National Institute of Standards and Technology, Gaithersburg, MD.

[22] J.H. Hubbell, Wm.J. Veigele, E.A. Briggs, R.T. Brown, D.T. Cromer and R.J. Howerton, Atomic form factors, incoherent scattering functions, and photon scattering cross sections, J. Phys. Chem. Ref. Data, 4 (1975) 471-538.

[23] D.E. Peplow and K. Verghese, Measured molecular coherent scattering form factors of animal tissues, plastics and human breast tissue, Phys. Med. Biol. 43 (1998) 2431-2452.

[24] J. Persliden and G. Alm Carlsson, Calculation of the small-angle distribution of scattered photons in diagnostic radiology using a Monte Carlo collision density estimator, Med. Phys. 13 (1986) 19-24.

[25] R. Brun, A. Gheata and M. Gheata The ROOT geometry package, Nucl. Inst. and Meth. in Phys. Res. A 502 (2003) 676-680.

[26] R.L. Siddon, Fast calculation of the exact radiological path for a threedimensional CT array, Med. Phys. 12 (1985) 252-255.

[27] A.J. Walker, An efficient method for generating discrete random variables with general distributions, ACM Trans. Math. Software, 3 (1977) 253 - 256.

[28] K. Cranley, B.J. Gilmore, G.W.A. Fogarty and L. Desponds, Catalogue of diagnostic x-ray spectra and other data, Report 78, Institute of Physics and Engineering in Medicine, 1997.

[29] R. Birch and M. Marshall, Computation of bremsstrahlung x-ray spectra and comparison with spectra measured with a Ge(Li) detector, Phys. Med. Biol. 24 (1979) 505-517.

[30] ICRU, Fundamental quantities and units for ionizing radiation, Report 60, ICRU, 1998.

[31] J. Sempau, A. Sanchez-Reyes, F. Salvat, H.O. ben Tahar, S.B. Jiang and J.M. Fernandez-Varea, Monte Carlo simulation of electron beams from an accelerator head using PENELOPE, Phys. Med. Biol. 46 (2001) 1163-1186.

[32] ICRU, Determination of dose equivalents from external radiation sources - part 2, Report 43, ICRU, 1988. 
[33] A. Malusek, M. Sandborg and G. Alm Carlsson, Calculation of the energy absorption efficiency function of selected detector arrays using the MCNP code, ISRN ULI-RAD-R-103-SE, 2007. Available on http://huweb.hu.liu.se/inst/imv/radiofysik/publi/rap.html.

[34] H.P. Chan and K. Doi, Energy and angular dependence of x-ray absorption and its effect on radiographic response in screen-film systems, Phys Med Biol, 28 (1983) 565-579.

[35] W.H. Press, W.T. Vetterling, S.A. Teukolsky and B.P. Flannery, Numerical recipes in C: the art of scientific computing (Cambridge University Press, 1992).

[36] C. Greskovich and S. Duclos, Ceramic scintillators, Annual review of materials science 27 (1997) 69-88.

[37] C.W. van Eijk, Inorganic scintillators in medical imaging, Phys. Med. Biol. 47 (2002) R85-106.

[38] G.J. Day and D.R. Dance, X-ray transmission formula for antiscatter grids, Phys. Med. Biol. 28 (1983) 1429-1433.

[39] L.R. Morin, Molecular form factors and photon coherent scattering cross sections of water, J. Phys. Chem. Ref. Data 11 (1982) 1091-1098.

[40] J. H. Siewerdsen and D. Jaffray, Cone-beam computed tomography with a flat-panel imager: Magnitude and effect of x-ray scatter, Med. Phys. 28 (2001) $220-231$.

[41] A. Malusek, M. Sandborg and G. Alm Carlsson, Validation of the CTmod toolkit, ISRN ULI-RAD-R-104-SE, 2007. Available on http://huweb.hu.liu.se/inst/imv/radiofysik/publi/rap.html. 